Corresponding authors:

Dana N Mitzel

USDA, Agricultural Research Service

Center for Grain and Animal Health Research

1515 College Ave.

Manhattan, KS 66502

(785)-537-5540

dana.mitzel@usda.gov
Juergen A. Richt

Kansas State University

College of Veterinary Medicine

1800 Denison Ave

Manhattan, KS, 66506

(785) $-532-2793$

jricht@ksu.edu

\title{
Susceptibility of midge and mosquito vectors to SARS-CoV-2 by natural route of infection
}

Velmurugan Balaraman $^{\mathrm{a}}$, Barbara S. Drolet ${ }^{\mathrm{b}}$, Natasha N Gaudreault ${ }^{\mathrm{a}}$, William C. Wilson ${ }^{\mathrm{b}}$, Jeana Owens $^{\mathrm{b}}$, Dashzeveg Bold ${ }^{\mathrm{a}}$, Dustin A. Swanson ${ }^{\mathrm{b}}$, Dane C. Jasperson ${ }^{\mathrm{b}}$, Leela E. Noronha ${ }^{\mathrm{b}}$, Juergen A. Richt ${ }^{a^{*}}$, Dana N Mitzel ${ }^{b^{*}}$

${ }^{a}$ Department of Diagnostic Medicine/Pathobiology and Center of Excellence for Emerging and Zoonotic Animal Diseases, College of Veterinary Medicine, Kansas State University, 1800 Denison Ave, Manhattan, Kansas, 66506, United States of America.

${ }^{b}$ United States Department of Agriculture, Agricultural Research Service, Arthropod-Borne Animal Diseases Research Unit, 1515 College Ave, Manhattan, Kansas, 66502, United States of America. 


\section{Abstract}

1 SARS-CoV-2 is a recently emerged, highly contagious virus and the cause of the current

2 pandemic. It is a zoonotic virus, although its animal origin is not clear yet. Person-to-person

3 transmission occurs by inhalation of infected droplets and aerosols, or by direct contact with

4 contaminated fomites. Arthropods transmit numerous viral, parasitic, and bacterial diseases;

5 however, the potential role of arthropods in SARS-CoV-2 transmission is not fully understood.

6 Thus far, a few studies have demonstrated that SARS-CoV-2 replication is not supported in cells

7 from certain insect species nor in certain species of mosquitoes after intrathoracic inoculation. In

8 this study, we expanded the work of SARS-CoV-2 susceptibility to biting insects after ingesting

9 a SARS-CoV-2infected blood meal. Species tested included Culicoides sonorensis biting

10 midges, as well as Culex tarsalis and Culex quinquefasciatus mosquitoes, all known biological

11 vectors for numerous RNA viruses. Arthropods were allowed to feed on SARS-CoV-2 spiked

12 blood and at various time points post infection analyzed for the presence of viral RNA and

13 infectious virus. Additionally, cell lines derived from C. sonorensis (W8a), Ae. aegypti (C6/36),

14 Cx. quinquefasciatus (HSU), and Cx. tarsalis (CxTrR2) were tested for SARS-CoV-2

15 susceptibility. Our results indicate that none of the biting insects, nor the insect cell lines support

16 SARS-CoV-2 replication. We conclude, that biting insect do not pose a risk for transmission of

17 SARS-CoV-2 to humans or animals following a SARS-CoV-2 infected blood meal.

18 Keywords: SARS-CoV-2, susceptibility, midges, mosquitoes 


\section{Introduction}

22 Severe acute respiratory syndrome coronavirus 2 (SARS-CoV-2) is the causative agent of the

232019 coronavirus disease (COVID-19) pandemic. SARS-CoV-2 belongs to the order

24 Nidovirales, family coronaviridae, and genus betacoronavirus. It is an enveloped virus with a

25 positive-sense, single-stranded RNA genome of approximately $30 \mathrm{~kb}$ in length (Chen et al.

26 2020). SARS-CoV-2 infects humans, and has the potential to infect various animal species (Chu

27 et al. 2020). Transmission from these animals to humans is not yet clearly understood. The virus

28 is mainly transmitted from person-to-person by inhalation of droplets and aerosols produced by

29 infected people (Chan et al. 2020), or through contact with contaminated surfaces (Goldman

30 2020, Sonja A. Rasmussen 2020; Kwon et al., 2020). Arthropods transmit numerous diseases to

31 humans and animals via biological and mechanical transmission (Leitner et al. 2015). Although

32 the SARS-CoV-2-related coronaviruses SARS-CoV-1 and MERS-CoV are not transmitted by

33 insects, concerns have been raised, by those in both public health and agricultural sectors, as to

34 their potential role in spreading SARS-CoV-2 among humans and animals. For arthropods to be

35 transmission-competent vectors, the respective pathogen must be acquired from a host during

36 blood feeding, then infect the midgut, escape the midgut barrier, disseminate to and infect the

37 salivary glands, and finally be transmitted to a susceptible host during subsequent blood feeding

38 (Franz et al. 2015). A recent report demonstrated that SARS-CoV-2 replication was not

39 supported in Aedes aegypti, Ae. albopictus and Culex quinquefasciatus mosquito species after an

40 intrathoracic route of infection (Huang et al. 2020). Another report showed that the SARS-CoV-

412 does not replicate in cells derived from Aedes mosquitoes, nor was it present in field-caught

42 Culex and Anopheles mosquitoes from Wuhan (Xia et al. 2020). 
43 Here, we report the first susceptibility study of SARS-CoV-2 infection using three critical insect

44 vectors following ingestion of a SARS-CoV-2 infected blood meal, including an agriculturally

45 important animal disease vector, Culicoides sonorensis biting midges, and two significant human

46 disease vector mosquito species, $C x$. tarsalis and $C x$. quinquefasciatus. Additionally, four insect-

47 derived cell lines from $C$. sonorensis (W8a), Ae. aegypti (C6/36), Cx. tarsalis (CxTrR2), and CX.

48 quinquefasciatus (HSU) were also evaluated for SARS-CoV-2 susceptibility.

\section{Methods}

49 The SARS-CoV-2 USA-WA1/2020 strain was acquired from Biodefense and Emerging

50 Infection Research Resources Repository (BEI Resources, Manassas, VA, USA) and was

51 passaged three times on VeroE6 cells (ATCC, VA, USA) with a final titer of $2.5 \times 10^{6}$

$52 \mathrm{TCID}_{50} / \mathrm{ml}$. Arthropod cell cultures were derived from C. sonorensis embryos (W8a; McHolland

53 and Mecham 2003), Cx. tarsalis embryos (CxTrR2; Arthropod-Borne Animal Diseases Unit;

54 ABADRU, Manhattan, KS, USA), Cx. quinquefasciatus ovaries (HSU; Hsu et al. 1970), and Ae.

55 albopictus larva (C6/36). The W8a, CxTrR2, HSU, and C6/36 cells were maintained in CuVa

56 medium, L-15 medium (with 10\% tryptose phosphate broth) and Medium 199H, respectively.

57 All media (Sigma-Aldrich, St. Louis, MO, USA) was supplemented with 10-20\% FBS (ITFBS;

58 Sigma). Cells were maintained at $27^{\circ} \mathrm{C}$ in closed T-flasks and inoculated with SARS-CoV-2 at

59 approximately 0.1 multiplicity of infection (MOI) for $1 \mathrm{~h}$ before the inoculum was replaced with

60 fresh culture media. Cell cultures were monitored for cytopathic effect (CPE) by light

61 microscopy and culture supernatants were collected at $0,2,4$, and 8 days post infection (dpi) for

62 subsequent titration by $\mathrm{TCID}_{50} \mathrm{CPE}$ assay on VeroE6 cells.

63 Cx. tarsalis, Cx. quinquefasciatus, and the ABADRU C. sonorensis colonies were reared and

64 maintained in the ABADRU insectary. Arthropods were transported to Kansas State University, 
Biosecurity Research Institute (BRI) for infection studies under Arthropod Containment Level-3

66

67 (ACL-3) conditions.

Adult female $C$. sonorensis $(\mathrm{n}=200)$ midges were allowed to feed on defibrinated sheep blood mixed 1:1 (v/v) with SARS-CoV-2 $\left(2.0 \times 10^{6} \mathrm{TCID}_{50} / \mathrm{ml}\right)$. Negative control unfed midges $(\mathrm{n}=100)$ were maintained in adjacent cages. For mosquitoes, 8-day old $C x$. tarsalis $(\mathrm{n}=100)$ or 10-day old $C x$. quinquefasciatus $(\mathrm{n}=100)$ were allowed to feed on SARS-CoV-2 spiked sheep blood as described above. Negative control mock-infected blood-fed $C x$. tarsalis $(\mathrm{n}=50)$ were maintained in adjacent cages. After an hour of feeding, midges or fully engorged mosquitoes were held at $28^{\circ} \mathrm{C}$ for 10 days. Surviving midges and mosquitoes at day 10 were pooled $(\mathrm{n}=5-10)$ in $1 \mathrm{ml}$ virus transport media (199E media supplemented with antibiotic-antimycotics; Sigma), and stored at $-80^{\circ} \mathrm{C}$ until processed for virus isolation (VI) and RNA extractions.

Pooled arthropods were homogenized by a Tissuelyser II (Qiagen, Germantown, MD, USA) using tungsten carbide beads (Qiagen). An aliquot (140 $\mu$ l) of homogenate was used for RNA extraction with the remaining homogenate filtered through a $0.22 \mu \mathrm{m}$ PES membrane filter (MIDSCI, St. Louis, MO, USA) before subsequent VI.

RNA extraction was performed using the QIAamp viral RNA mini kit (Qiagen) as per manufacturer's instructions. RT-qPCR assay was performed according to the Center for Disease Control (CDC) protocol for detection of SARS-CoV-2 nucleocapsid (N)-specific RNA

\section{(https://www.fda.gov/media/134922/download) using Script XLT One-Step RT-qPCR Tough}

Mix (Quanta Biosciences, Beverly, MA, USA) on a CFX96 Real-time thermocycler (BioRad, Hercules, CA, USA). Plate controls included a quantitated SARS-CoV-2 N-specific qPCR positive control, diluted 1:10 (Integrated DNA Technologies, IA, USA), and a non-template 
87 control (NTC). Results were analyzed using the Bio-Rad CFX Manager 3.1 with samples below

$88 \quad 40 \mathrm{Ct}$ considered positive.

89 Arthropod homogenates $(100 \mu \mathrm{l})$ were added on to VeroE6 cells in 24 well plates and incubated

90 at $37^{\circ} \mathrm{C}$ and $5 \% \mathrm{CO}_{2}$ for 3 days. Culture supernatents were blind-passaged three times on VeroE6

91 cells, and at the first and third passage, cells were examined by an indirect immunofluorescence

92 assay (IFA) for the presence of SARS-CoV-2 antigen. Briefly, 3 dpi cells were fixed with ice

93 cold $100 \%$ methanol for 10 mins at $-80^{\circ} \mathrm{C}$ and washed three times with $1 \times$ PBS Tween 20

$94(0.05 \%)$. Mouse monoclonal antibodies (in house) specific for the Receptor Binding Domain

95 (RBD) of spike protein of SARS-CoV-2 was diluted 1:5 in 1x PBS containing 1\% BSA and 150

$96 \mu \mathrm{l}$ was added to each well and incubated at room temperature (RT) for $1 \mathrm{~h}$. The cells were

97 washed three times as described above, and then incubated with $150 \mu$ l of FITC-conjugated goat

98 anti-mouse IgG (Abcam, Cambridge, MA, USA), diluted 1:500 in 1x PBS with BSA, for $1 \mathrm{~h}$ at

99 RT. After washing and drying, cell monolayers were examined by an EVOS fluorescent

100 microscope (ThermoFisher Scientific, Waltham, MA, USA) for the presence of FITC positive

101 cells. Mock infected and SARS-CoV-2 infected VeroE6 cells were used as negative and positive

102 controls, respectively.

\section{Results and discussion}

103 The goal of this study was to determine whether arthropods are susceptible to SARS-CoV-2 by a

104 natural route of infection which has not yet been evaluated. In addition to important mosquito

105 vectors, Culicoides midges were also evaluated in this study. Initial infection studies were

106 performed in vitro with the insect cell lines W8a, C6/36, CxTrR2, and HSU. Two independent

107 experiments showed no obvious sign of CPE for any of the SARS-CoV-2-infected arthropod- 
108

109

derived cell cultures, nor for any of the insect culture supernatants collected at 2, 4 or 8 dpi and passaged on VeroE6 cells.

Next, susceptibility of insects after an infectious blood meal was investigated. Of 200 midges allowed to feed on the SARS-CoV-2-spiked blood meal, 140 survived until 10 days post blood meal and were further analyzed. The majority $(85 \%)$ of virus-fed midge pools had detectable SARS-CoV- 2-specific RNA with an average Ct value of 34.84 \pm 2.6 ; the day 10 control unfed midges were negative for SARS-CoV-2 RNA (Table 1). Among the Cx. tarsalis mosquitoes allowed to feed on SARS-CoV-2-spiked blood ( $\mathrm{n}=100)$, only 48 virus-fed mosquitoes survived until day 10. One out of 6 (17\%) virus-fed $C x$. tarsalis mosquitoes had detectable SARS-CoV-2specific RNA with an average $\mathrm{Ct}$ value of 31.3, and none of the 30 mock-infected blood-fed $C x$. tarsalis mosquitoes were SARS-CoV-2 RNA positive (Table 1). Similarly, of $100 C x$. quinquefasciatus mosquitoes, 47 virus-fed survived until day 10. Viral RNA was detected in $50 \%$ of the SARS-CoV-2-fed mosquitoes with an average $\mathrm{Ct}$ value of 34.17 (Table 1).

To determine the presence of infectious virus, serial passages of pooled arthropod homogenates were performed on VeroE6 cells. No CPE was observed after three passages of virus-fed Culicoides midge homogenates, and IFA analysis of passage one and three of inoculated VeroE6 cells confirmed the absence of SARS-CoV-2 (Table 2; Figure 1). SARS-CoV-2-infected VeroE6 cells were used as an IFA positive control and showed a clear positive staining pattern (Fig.1). Unfed control midge samples were negative by VI and IFA (Table 2). Similarly, no infectious virus was detected from any of the six homogenate pools of SARS-CoV-2-fed Cx. tarsalis mosquitoes that were passaged on VeroE6 cells and analyzed for CPE and by IFA; the control Cx. tarsalis homogenate pools were also negative by both methods (Table 2). The six virus-fed 
CX. quinquefasciatus mosquito homogenates tested for infectivity by VI and IFA were also negative for SARS-CoV-2 (Table 2).

A key factor crucial for arthropod-mediated transmission is that the infected host, either person or animal, is viremic at the time of feeding. Thus far, SARS-CoV-2 is known to cause viremia in some cases of infected people (Young et al. 2020). Susceptible animal models tested so far appear to be aviremic (Shi et al. 2020) except for hamsters which regularly show viremia (Chan et al. 2019). Therefore, controlled studies to rule out arthropod transmission of this RNA virus are critical for determining risk, as well as developing accurate epidemiological modeling and control strategies.

Overall, our results agree with previously published findings that Aedes mosquito derived cells do not support SARS-CoV-2 replication (Xia et al. 2020). Additionally, we have shown that two different Culex species derived cell lines and one Culicoides midge derived cell line are also refractory to SARS-CoV-2 infection. In a previously published SARS-CoV-2 susceptibility study in mosquitoes, intrathoracic injection of SARS-CoV-2 grown in Vero76 was used to determine the susceptibility of Ae. aegypti, Ae. Albopictus, and Cx. quinquefasciatus to the virus (Huang et al. 2020); however, intrathoracic inoculation bypasses the natural route of infection via the ingestion of a virus-infected blood meal (Franz et al. 2015). Therefore, in the present study, testing of arthropods for SARS-CoV-2 susceptibility was performed following the ingestion of a virus-spiked blood meal; this is important not only because it is the most relevant route of infection for arthropod vectors, but also because of the quasi-species nature of an RNA virus inoculum such as for SARS-CoV-2 (Jary et al. 2020). Considerable genetic bottle necks and natural selection processes exist for viruses in arthropod replication and arthropod-borne transmission. Depending on the insect vector, the number of virus particles ingested via a blood 
153 meal depends on the level of viremia in the host and the volume of the meal. When virus

154 particles ingested via the blood meal enter the midgut, few will be able to infect the midgut

155 epithelium. After midgut replication, progeny viruses escape the midgut barrier and disseminate

156 in the insect's hemocoel, and then next they infect and replicate in the salivary gland. This is

157 critical for subsequent virus transmission to a susceptible host during a blood meal. The quasi-

158 species nature of RNA viruses combined with the natural selection for defined viral genotypes

159 enables RNA viruses to infect and replicate in arthropods and results in a defined virus

160 population selected for increased fitness for the arthropod environment. These viral genotypic

161 changes could lead to viral biotypes with a higher ability of salivary gland infection and bite

162 transmission, when compared to a virus which is artificially injected into the insect's hemocoel.

163 Our in vitro and in vivo studies of midge and mosquito susceptibility to SARS-CoV-2 infection

164 following a natural ingestion route of exposure showed that viral RNA remained in virus-fed

165 arthropods for up to 10 days post virus-spiked blood feeding. However, no infectious virus was

166 recovered from these RNA-positive arthropods, even after three passages on highly susceptible

167 VeroE6 cells. Our in vitro studies using various insect cells support these results since no

168 infectious virus was detected in supernatants of SARS-CoV-2 inoculated insect cell cultures. In

169 conclusion, the insect vector species known to transmit animal and human pathogens used in this

170 study are refractory to SARS-CoV-2 infection under experimental conditions and, therefore,

171 most likely do not play a role in transmission of SARS-CoV-2.

172 Acknowledgments

173 We gratefully thank the staff of KSU Biosecurity Research Institute. The following reagent was

174 obtained through BEI Resources, National Institute of Allergy and Infectious Diseases (NIAID),

175 National Institutes of Health (NIH): SARS-CoV-2 Virus strain USA-WA1/2020 (catalogue \# 
176 NR-52281). C6/36 cells were kindly provided by Robert B Tesh, UTMB, Galveston, TX.Cx.

177 tarsalis and Cx. quinquefasciatus colonies were kindly provided by Chris Barker and O.

178 Winokur, University of California-Davis.

179 Conflict of interest

180 All the authors declare no conflict of interest.

\section{Disclaimer}

182 The conclusions in this report are those of the author and do not necessarily represent the views

183 of the United States Department of Agriculture.

$184 \quad$ Funding

185 Funding for this study was in part by the United States Department of Agriculture (BSD, DM,

186 DS, JO, DCJ, LN, and WCW) and through grants from NBAF Transition Funds and KSU

187 internal funds to JAR. This study was also partially supported by NIAID Centers of Excellence

188 for Influenza Research and Surveillance (CEIRS; contract \#HHSN 272201400006C), and the

189 Department of Homeland Security Center of Excellence for Emerging and Zoonotic Animal

190 Diseases (grant \#2010-ST061-AG0001) to JAR. 


\section{References}

Chan, J. F. W., S. Yuan, K. H. Kok, K. K. W. To, H. Chu, J. Yang, F. Xing, J. Liu, C. C. Y. Yip, R. W. S. Poon, H. W. Tsoi, S. K. F. Lo, K. H. Chan, V. K. M. Poon, W. M. Chan, J. D. Ip, J. P. Cai, V. C. C. Cheng, H. Chen, C. K. M. Hui, and K. Y. Yuen. 2020. A familial cluster of pneumonia associated with the 2019 novel coronavirus indicating personto-person transmission: a study of a family cluster. Lancet. 395: 514-523.

Chan, J. F., A. J. Zhang, S. Yuan, and V. Kwok-. 2019. Title: Simulation of the clinical and pathological manifestations of Coronavirus Disease 2019 (COVID-19) in golden Syrian hamster model: implications for disease pathogenesis and transmissibility Authors: Jasper Fuk-Woo Chan. Clin Infect Dis. 2019: 1-50.

Chen, Y., Q. Liu, and D. Guo. 2020. Emerging coronaviruses: Genome structure, replication, and pathogenesis. J. Med. Virol. 92: 418-423.

Chu, H., J. F.-W. Chan, T. T.-T. Yuen, H. Shuai, S. Yuan, Y. Wang, B. Hu, C. C.-Y. Yip, J. O.-L. Tsang, X. Huang, Y. Chai, D. Yang, Y. Hou, K. K.-H. Chik, X. Zhang, A. Y.-F. Fung, H.-W. Tsoi, J.-P. Cai, W.-M. Chan, J. D. Ip, A. W.-H. Chu, J. Zhou, D. C. Lung, K.-H. Kok, K. K.-W. To, O. T.-Y. Tsang, K.-H. Chan, and K.-Y. Yuen. 2020. Comparative tropism, replication kinetics, and cell damage profiling of SARS-CoV-2 and SARS-CoV with implications for clinical manifestations, transmissibility, and laboratory studies of COVID-19: an observational study. The Lancet Microbe. 1: e14-e23.

Franz, A. W. E., A. M. Kantor, A. L. Passarelli, and R. J. Clem. 2015. Tissue barriers to arbovirus infection in mosquitoes. Viruses. 7: 3741-3767.

Goldman, E. 2020. Exaggerated risk of transmission of COVID-19 by fomites. Lancet Infect. Dis. 20: 892-893.

Hsu, S. H., W. H. Mao, and J. H. Cross. 1970. Establishment of a Line of Cells Derived from Ovarian Tissue of Culex Quinquefasciatus Say1. J. Med. Entomol. 7: 703-707.

Huang, Y. J. S., D. L. Vanlandingham, A. N. Bilyeu, H. M. Sharp, S. M. Hettenbach, and S. Higgs. 2020. SARS-CoV-2 failure to infect or replicate in mosquitoes: an extreme challenge. Sci. Rep. 10: 1-4.

Jary, A., V. Leducq, I. Malet, S. Marot, E. Klement-Frutos, E. Teyssou, C. Soulié, B. Abdi, M. Wirden, V. Pourcher, E. Caumes, V. Calvez, S. Burrel, A.-G. Marcelin, and D. Boutolleau. 2020. Evolution of viral quasispecies during SARS-CoV-2 infection. Clin. Microbiol. Infect.

Leitner, W. W., T. Wali, R. Kincaid, and A. Costero-Saint Denis. 2015. Arthropod Vectors and Disease Transmission: Translational Aspects. PLoS Negl. Trop. Dis. 9: 1-11.

Main, B. J., J. Nicholson, O. C. Winokur, C. Steiner, K. K. Riemersma, J. Stuart, R. Takeshita, M. Krasnec, C. M. Barker, and L. L. Coffey. 2018. Vector competence of Aedes aegypti, Culex tarsalis, and Culex quinquefasciatus from California for Zika virus. PLoS Negl. Trop. Dis. 12: 1-13. 
McHolland, L. E., and J. O. Mecham. 2003. Characterization of cell lines developed from field populations of Culicoides sonorensis (Diptera: Ceratopogonidae). J. Med. Entomol. 40: $348-351$.

Shi, J., Z. Wen, G. Zhong, H. Yang, C. Wang, B. Huang, R. Liu, X. He, L. Shuai, Z. Sun, Y. Zhao, P. Liu, L. Liang, P. Cui, J. Wang, X. Zhang, Y. Guan, W. Tan, G. Wu, H. Chen, Z. Bu, and Z. Bu. 2020. Susceptibility of ferrets, cats, dogs, and other domesticated animals to SARS-coronavirus 2. Science (80-. ). 368: 1016-1020.

Sonja A. Rasmussen, MD, MS, J. C. S. 2020. Since January 2020 Elsevier has created a COVID-19 resource centre with free information in English and Mandarin on the novel coronavirus COVID-. Ann Oncol. 19-21.

Kwon, T., Gaudreault, N. N, and Richt, J. A. 2020. Environmental stability of SARS-CoV-2 on different types of surfaces under indoor and seasonal climate conditions. bioRxiv.

Xia, H., E. Atoni, L. Zhao, N. Ren, D. Huang, R. Pei, Z. Chen, J. Xiong, R. Nyaruaba, S. Xiao, B. Zhang, and Z. Yuan. 2020. SARS-CoV-2 Does Not Replicate in Aedes Mosquito Cells nor Present in Field-Caught Mosquitoes from Wuhan. Virol. Sin. 35: 355-358.

Young, B. E., S. W. X. Ong, S. Kalimuddin, J. G. Low, S. Y. Tan, J. Loh, O. T. Ng, K. Marimuthu, L. W. Ang, T. M. Mak, S. K. Lau, D. E. Anderson, K. S. Chan, T. Y. Tan, T. Y. Ng, L. Cui, Z. Said, L. Kurupatham, M. I. C. Chen, M. Chan, S. Vasoo, L. F. Wang, B. H. Tan, R. T. P. Lin, V. J. M. Lee, Y. S. Leo, and D. C. Lye. 2020. Epidemiologic Features and Clinical Course of Patients Infected with SARS-CoV-2 in Singapore. JAMA - J. Am. Med. Assoc. 323: 1488-1494.

Zhu, N., D. Zhang, W. Wang, X. Li, B. Yang, J. Song, X. Zhao, B. Huang, W. Shi, R. Lu, P. Niu, F. Zhan, X. Ma, D. Wang, W. Xu, G. Wu, G. F. Gao, and W. Tan. 2020. A novel coronavirus from patients with pneumonia in China, 2019. N. Engl. J. Med. 382: 727-733. 
Table 1: Detection of SARS-CoV-2 viral RNA in various arthropods by RT-qPCR

\begin{tabular}{ccc}
\hline Treatment Group & $\begin{array}{c}\text { Positive homogenate } \\
\text { pools out of total }(\% \\
\text { positive })\end{array}$ & Mean Ct \pm SD \\
\hline Unfed C. sonorensis & $0 / 4(0)$ & ND $^{*}$ \\
SARS-CoV-2 fed C. sonorensis & $12 / 14(85)$ & $34.84 \pm 2.6$ \\
Mock-infected fed Cx. tarsalis & $0 / 3(0)$ & $\mathrm{ND}^{*}$ \\
SARS-CoV-2 fed Cx. tarsalis & $1 / 6(17)$ & 31.3 \\
SARS-CoV-2 fed C $x$. & $3 / 6(50)$ & $34.17 \pm 3.3$ \\
quinquefasciatus & & \\
\hline *ND=not detected & &
\end{tabular}

Table 2: Detection of infectious SARS-CoV-2 in arthropod homogenate pools on VeroE6 cells by virus isolation and an indirect immunofluorescence assay

\begin{tabular}{|c|c|c|}
\hline Species & Control arthropods & SARS-CoV-2 fed arthropods \\
\hline & $\begin{array}{c}\text { VI } \quad \text { IFA } \\
\text { (positive/N) }\end{array}$ & $\begin{array}{l}\text { VI } \begin{array}{r}\text { IFA } \\
(\text { positive/N) }\end{array}\end{array}$ \\
\hline C. sonorensis & $0 / 4 \quad 0 / 4$ & $0 / 14 \quad 0 / 14$ \\
\hline Cx. tarsalis & $0 / 3$ & $0 / 6$ \\
\hline Cx. quinquefasciatus & N/A N/A & $0 / 6$ \\
\hline
\end{tabular}

$\mathrm{N}=$ total number of day 10 homogenate pools. $0=$ no virus positive or FITC positive cell cultures; $\mathrm{N} / \mathrm{A}=$ not tested 
Fig. 1.

\section{SARS-CoV-2}

\section{homogenate}

\section{Bright field}

FITC filter

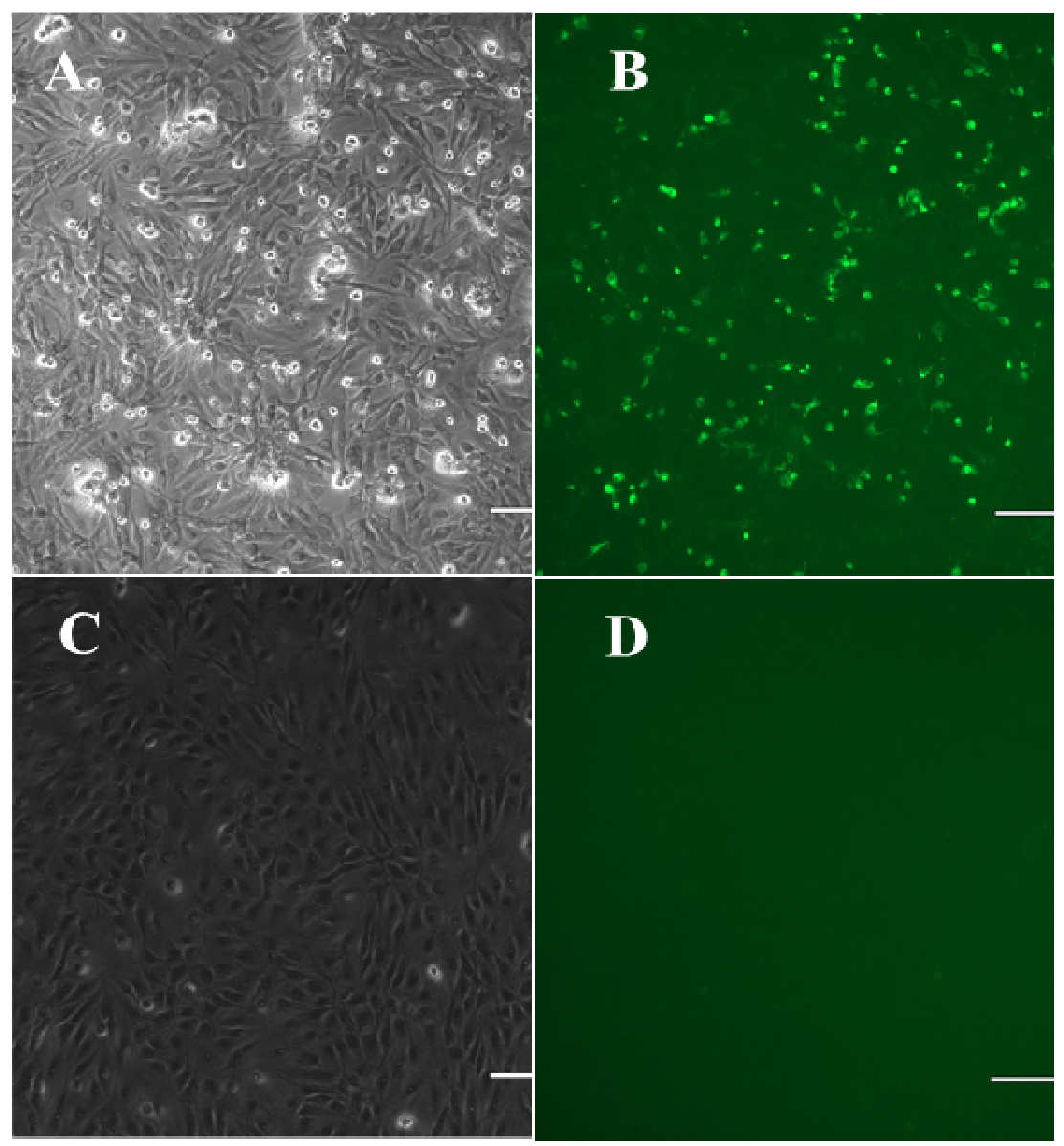

Figure 1. Indirect immunofluorescence assay for the detection of SARS-CoV-2 infected cells. SARS-CoV-2 and a representative passage 3 arthropod homogenate were incubated 3 days on VeroE6 cells. A) Bright field; B) Positive SARS-CoV-2 infected VeroE6 cells (positive control cells); and C) Bright field; D) VeroE6 cells inoculated with passage three of a RT-qPCR+Culicoides sonorensis day 10 homogenate.

(Magnification 10x) 